\title{
Análisis de la influencia percibida de la capacidad matemática en la elección de una carrera
}

\section{An analysis of the perceived influence of mathematical capacity in a degree election}

\author{
Sebastián Acosta Madiedo, Andrés Almeida Guerrero, Paula Arias Valenzuela, \\ Gabriela Garavito Munar, Nicolás López Contreras, Franklin Posos Ramos, \\ Angie Sibaja Jaramillo*
}

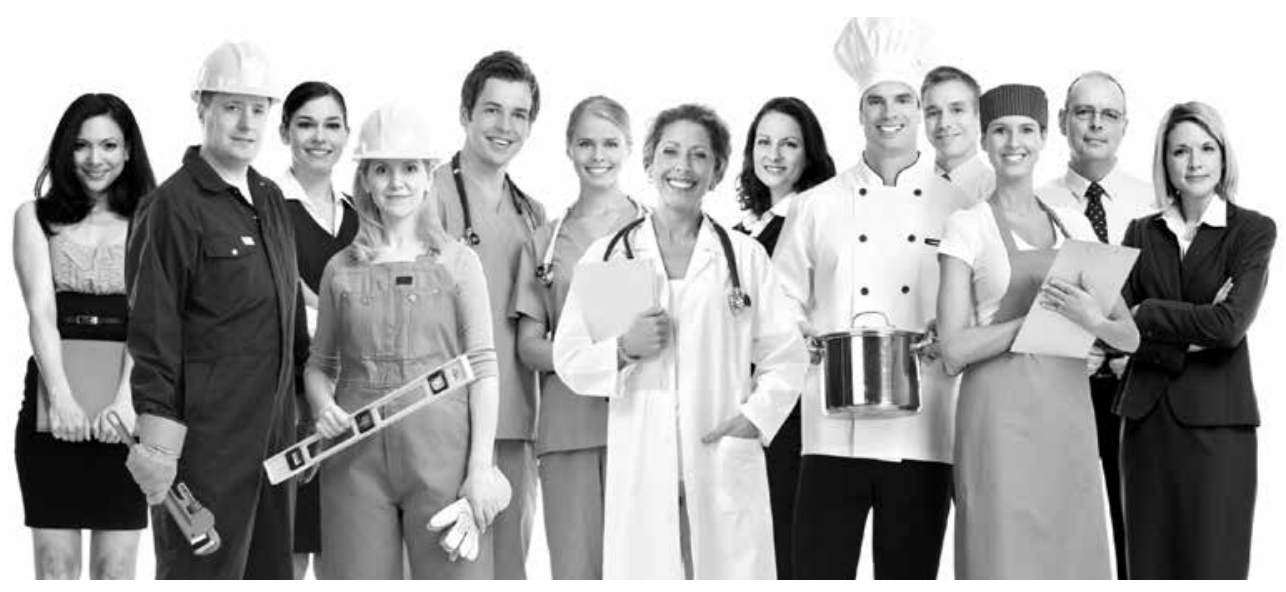

\section{Resumen}

La matemática se ha caracterizado como una de las áreas más complejas del conocimiento. En muchos casos se cree que esta área, por sí sola, ejerce una gran influencia sobre la carrera, o por lo menos, el área profesional en que se desempeñará un individuo al graduarse del colegio. El presente trabajo pretende investigar el nivel de correlación entre la escogencia de carrera de un individuo y su desempeño en matemáticas tomando como muestra un grupo de estudiantes de distintas facultades de la Universidad Nacional, a los cuales se les interroga acerca de su decisión de carrera y su desempeño en el área de matemáticas en la prueba de Estado Saber 11 (ICFES).

Palabras clave: influencia, matemáticas, Saber 11, elección, carrera.

Citar este artículo como: Acosta, S., Almeida, A., Arias, P., Garavito, G., López, N., Posos, F. y Sibaja, A. (2016). Análisis de la influencia percibida de la capacidad matemática en la elección de una carrera. Revista Papeles, 8(16), 86-98.

Fecha recibido: octubre 20 de 2016.

Fecha aprobado: diciembre 5 de 2016.

* Estudiantes de Economía de la Universidad Nacional de Colombia. Estudio llevado a cabo en el curso Metodología de la investigación, bajo la dirección del profesor Enrique Ferrer-Corredor. Correo electrónico: ggaravitom@unal.edu.co 


\begin{abstract}
Mathematics has been generally recognised as one of the most complex areas of knowledge. In many cases, people believe that a student's performance on this area exerts a great influence over the degree, or at least, the area of knowledge a student chooses to major once he graduates from High School. This academic paper intends to research the level of correlation between a student's degree choice and his performance in mathematics. The reference frame will be a group of students from different faculties of the Universidad Nacional de Colombia, who will be surveyed about the degree they are majoring in and their performance in Mathematics in the state exam "Saber 11" (ICFES).
\end{abstract}

Keywords: influence, mathematics, Saber 11, degree, choice.

\title{
Análisis de la influencia percibida de la capacidad matemática en la elección de una carrera
}

La elección de la profesión es un proceso arduo y meticuloso; las decisiones vocacionales se van tomando, de manera consciente o inconsciente, a medida que el aspirante se enfrenta a la compleja red de factores que debe tener en consideración. Algunos factores pesan más que otros, el individuo está siendo impulsado constantemente en una dirección u otra.

Quizá, al preguntarle a un estudiante por qué eligió su carrera, este empiece a profesar su amor por la carrera. Tal afirmación es válida, $\mathrm{y}$ en algunos casos la plena verdad, pero a menudo existen razones subyacentes, aunque nos percatemos de ellas o no, que como una brújula orientan nuestra dirección.
Aunque puede ser afectada por características personales, como la disciplina o ambición, es indudable que la aptitud de un individuo para desempeñarse en ámbitos determinados juega un rol crucial en la toma de esta decisión de este hito en la vida. Entre estos ámbitos conclusivos destacaremos la matemática, como una de las ramas más complejas del conocimiento.

Partiendo, entonces, desde los resultados obtenidos en el componente matemático del examen colombiano Saber 11 (ICFES) de los admitidos para el año 2015 a la Universidad Nacional, sede de Bogotá, esta investigación pretende responder a la pregunta: ¿cómo influye el nivel matemático de una persona en la elección de su carrera de pre-grado?

\section{Objetivo general}

Realizar un análisis comparativo entre el nivel matemático de los admitidos a siete facultades de la Universidad Nacional de Colombia según el examen colombiano Saber 11 (ICFES) y cómo este resultado incide en la escogencia de carrera de pre-grado de los alumnos.

\section{Objetivos específicos}

Identificar y contrastar la distribución del puntaje ICFES en, y entre, una selección de facultades en la Universidad Nacional de Colombia.

Distinguir tendencias entre la influencia percibida por los estudiantes del puntaje matemático (ICFES) sobre su elección de carrera.

Establecer una relación entre el puntaje ICFES y la propensión a escoger carreras alternativas. 


\section{Hipótesis iniciales}

Al iniciar esta investigación se tiene como primera hipótesis que, en general, existe una relación directa y proporcional entre el nivel matemático del aspirante y la complejidad convencionalmente reconocida del componente de matemática en la carrera elegida. Esto se debe a que el aspirante tiende a gravitar hacia la carrera para la cual se considera más apto por naturaleza, ya que es aquella donde se siente más propenso a tener éxito. Por esta razón se espera que el nivel matemático del admitido (medido por el examen Saber 11) influya con una mayor fuerza para las carreras convencionalmente vistas como orientadas hacia las matemáticas, y con menor fuerza para las carreras típicamente vistas como 〈ajenas〉 a este rama del conocimiento.

Como segunda hipótesis, se plantea que a menor puntaje en el examen Saber 11, mayor la probabilidad de tener carreras alternativas. Esto se explica por el alto nivel de competencia en la Universidad Nacional de Colombia. Si un aspirante obtuvo un puntaje bajo en el ICFES, la probabilidad de sacar un puntaje suficiente en el examen de ingreso a la Nacional disminuye, por lo que el aspirante decidido a ingresar a la universidad debe contar con una carrera alternativa donde la probabilidad de ser admitido sea mayor.

\section{Marco teórico}

Ante todo, para poder realizar esta investigación es necesario definir un marco teórico dentro del cual establecer la investigación. En este sentido, se definirán unos conceptos elementales a la investigación y se especificarán los parámetros de esta.

\section{Conceptos básicos}

El Saber 11 es un examen de evaluación a la educación media creado por el ICFES (Instituto Colombiano para el Fomento de la Educación Superior). Esta prueba, más reconocida como "ICFES", se aplica a los estudiantes que se encuentran cursando su último año de bachillerato para determinar su nivel y capacidad en distintas áreas de formación. El examen está dividido en cinco componentes: lectura crítica, matemáticas, ciencias naturales, sociales y competencias ciudadanas, e inglés, siendo 100 la calificación máxima por sección.

Esta prueba no solo fue creada para medir la capacidad de los estudiantes, sino que es de carácter obligatorio para acceder a la educación superior en cualquier universidad nacional. A pesar de existir diversos criterios para ingresar a la educación superior, como los son las entrevistas, los exámenes de conocimientos y puntaje global que arrojan estos exámenes son el filtro que usan varias instituciones de educación superior para admitir a los aspirantes. De este modo, entre mayor sea el puntaje, mayor la probabilidad tienen los estudiantes de ser aceptados dentro de la universidad de preferencia. Las carreras más demandadas (Cégep, 2012), como lo son medicina, ingeniería de sistemas, geología, y arquitectura, requieren un mayor puntaje, por lo que un gran porcentaje de sus aspirantes no logran entrar la carrera y/o universidad que habían elegido como primera opción.

Por otra parte, cabe resaltar que la Universidad Nacional de Colombia cuenta con su propio examen de admisión, el cual evalúa competencias similares, agregando un componente de análisis de imagen, y a su vez excluyendo inglés, competencias ciudadanas y sociales. Es relevante advertir, por lo tanto, que la Universidad Nacional de Colombia no tiene 
en consideración el resultado del aspirante en el examen nacional ICFES. No obstante, para efectos prácticos, se trabaja con la suposición de que el individuo ha seleccionado una carrera de preferencia antes de aplicar a la Universidad.

\section{Delimitación y limitaciones de la investigación}

La presente investigación considera únicamente los ingresados a las facultades de Artes, Ciencias, Ciencias Económicas, Ciencias Humanas, Derecho, Ciencias Políticas y Sociales, Enfermería, e Ingeniería.

Esta investigación, a juicio de sus autores, cuenta con tres limitaciones principales.

En primer lugar, las limitaciones derivadas de la inconsciencia personal en el proceso de toma de decisiones. Es decir, partimos del hecho hipotético de que para tomar la mayoría de decisiones cotidianas, un individuo no dedica gran parte de su tiempo. Decisiones que se han vuelto un hábito, como tener un color favorito, se vuelven connaturales a la persona (Ortiz y Zabala, 2005). De un modo similar, pero a mayor escala, la misma lógica aplica en decisiones como la carrera que estudiarás. Una persona con aptitud natural para las matemáticas gravitará hacia disciplinas donde este componente sea significativo y/o ventajoso para ella (Ortiz y Zabala, 2001). En este orden de ideas, es posible y probable que al momento de reflexionar sobre factores influyentes en la escogencia de carrera se desconozcan decisiones inconscientes, como preferencia por una disciplina derivada de la aptitud natural por ella.
La segunda limitación, fuertemente ligada a la anterior, se sintetiza en que un individuo toma en consideración una gran variedad de factores al momento de tomar una decisión, usualmente orientado a racionalizar el problema y tomar la decisión de mayor beneficio para él. Existirán siempre las decisiones "irracionales" o fundamentadas en factores aun no concretados, como lo son las emociones (Harkins \& Singer, 2009). El peso de un factor no es homogéneo en todos los casos, por lo cual se debe trabajar bajo la suposición de priorización de factores, donde aquellos más 'racionales' ocupan un nivel jerárquico superior.

Por último, la muestra seleccionada de la población total (siendo la población todos los alumnos de las facultades previamente mencionadas) es de un tamaño significativamente reducido. Esto se debe a los múltiples cambios que ha tenido el sistema de calificación del examen Saber 11 en su escala de calificación, que implica la necesidad de restringir la muestra útil a aquellos calificados bajo un sistema homogéneo. Para efectos de investigación se consideró únicamente los admitidos con resultados en el Saber 11 evaluados sobre 100 .

\section{Metodología}

La metodología utilizada para esta investigación fue en su mayoría cuantitativa (Hernández, et al. 2014). Los datos se recolectaron con la colaboración de estudiantes, elegidos al azar, pertenecientes de distintas facultades. Estos fueron cuestionados respecto a su escogencia de carrera, su interés de realizar un doble programa y el nivel de influencia que, según ellos, la prueba de estado tuvo sobre su decisión de carrera, brindando cuatro categorías escalando el nivel de influencia. A partir de estas preguntas se realizó una tabulación de las encuestas que brindaron datos cuantitativos acerca de la 
correlación que existe entre los resultados en la sección de matemáticas de la prueba de estado y la escogencia de carrera de cada uno de los individuos encuestados.

El presente trabajo muestra las tabulaciones de cada uno de los datos de la encuesta por facultad y en conjunto, y establece las correlaciones pertinentes (o la falta de correlación) de manera escrita.

\section{Análisis por facultades}

Tabla 1. Ciencias

\begin{tabular}{|l|l|c|}
\hline Facultad & $\begin{array}{l}\text { Carreras a las } \\
\text { que se le aplicó la } \\
\text { encuesta }\end{array}$ & $\begin{array}{c}\text { Número de } \\
\text { encuestas } \\
\text { realizadas }\end{array}$ \\
\hline Ciencias & $\begin{array}{l}\text { Matemáticas, } \\
\text { Estadística, Física, } \\
\text { Química, Biología. }\end{array}$ & 52 \\
\hline
\end{tabular}

Fuente: elaboración propia.

En la Facultad de Ciencias se realizaron encuestas en 52 personas. En esta facultad se encuentran los pre-grados de biología, estadística, farmacia, física, matemáticas y química. A continuación se presentarán las gráficas que arrojaron las encuestas y su análisis respectivo

Gráfica 1. Número de personas en cada intervalo de puntaje (Ciencias)

Distribución de puntaje de ICFES

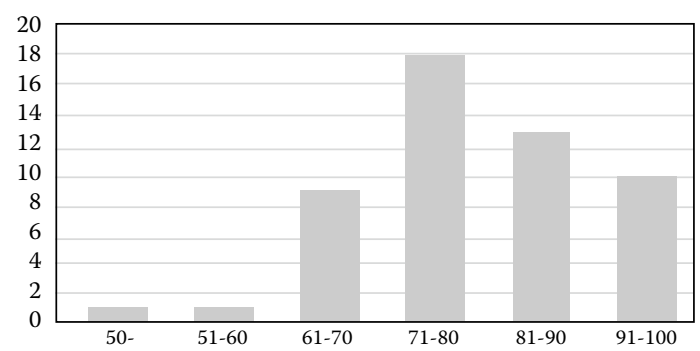

Fuente: elaboración propia.

Como era de esperarse, la Facultad de Ciencias de la Universidad Nacional, sede Bogotá, tiene en general un mayor puntaje que el resto de la universidad en el componente matemático.
Gráfica 2. Tendencia de influencia por puntaje (Ciencias)

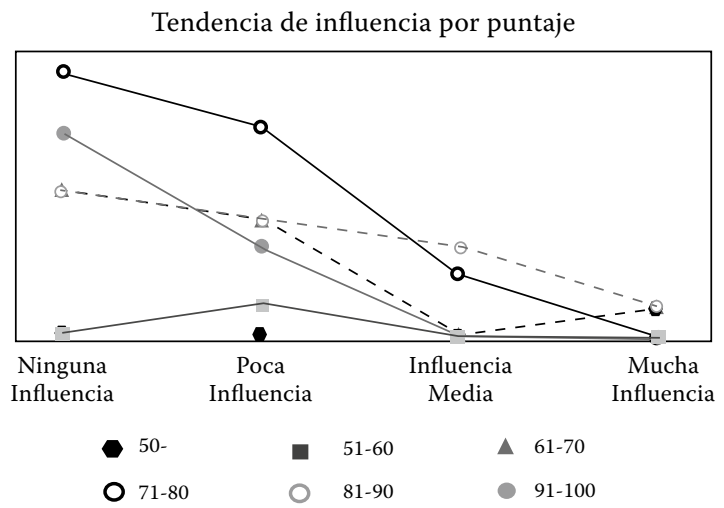

Fuente: elaboración propia.

Al momento de relacionar el puntaje con la influencia, se sigue la misma tendencia que en general: para la mayoría de los estudiantes, el ICFES no tuvo mayor influencia en su decisión de carrera, independientemente del resultado que obtuvieron. En la de facultad ciencias se puede ver una pequeña tendencia a que entre más puntaje se tenga, los encuestados dicen tener menos influencia del puntaje, de manera que el mayor número de personas que no proclamaron no tener ninguna influencia se encuentran en el intervalo de puntajes de 91-100.

Gráfica 3: Tendencia de opción alternativa previa a la realización del examen Saber 11 en relación al puntaje (Ciencias)

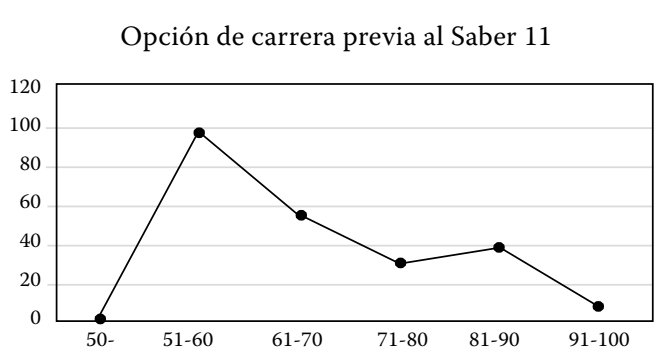

Fuente: elaboración propia.

Esta gráfica muestra una relación negativamente correlativa entre el puntaje y la opción inicial de carrera. De esto se puede inferir una causación del puntaje ICFES al momento de 
decidir la carrera final. Por último, en esta facultad hay una relación clara: entre más puntaje, disminuye el número de personas con otra opción de carrera. Los autores interpretamos esto como que en la facultad de ciencias, debido al alto componente matemático de los pre-grados que se encuentran aquí, un alto puntaje en el componente matemático da más seguridad a los estudiantes en cuanto a su decisión de carrera.

Tabla 2. Artes

\begin{tabular}{|c|c|c|}
\hline Facultad & $\begin{array}{c}\text { Carreras a las que } \\
\text { se le aplicó la } \\
\text { encuesta }\end{array}$ & $\begin{array}{c}\text { Número } \\
\text { de encuestas } \\
\text { realizadas }\end{array}$ \\
\hline Artes & Diseño industrial & 32 \\
\hline
\end{tabular}

Fuente: elaboración propia.

En la Facultad de Artes se realizaron 32 encuestas a estudiantes de diseño industrial de los primeros cuatro semestres de su carrera. A continuación se presentarán las gráficas que arrojaron las encuestas y el análisis respectivo de cada una:

Gráfica 4. Número de personas en cada intervalo de puntaje (Artes)

Distribución de puntaje de la prueba Saber 11

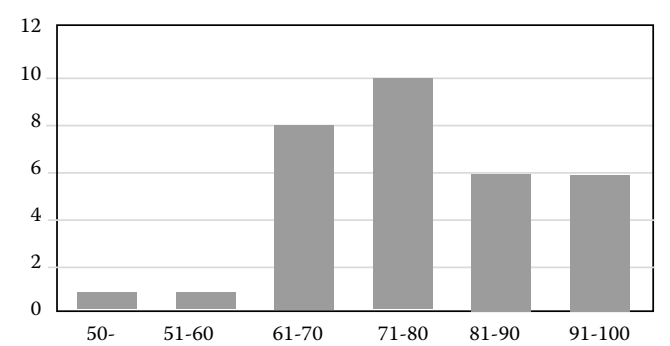

Fuente: elaboración propia.

A diferencia de lo esperado, el puntaje obtenido por los estudiantes de la Facultad de Artes en el componente de matemáticas tiende a ubicarse en los rangos más altos, comparables con facultades como la de ciencias e ingeniería.
Gráfica 5. Tendencia de influencia por puntaje (Artes)

Tendencia de influencia por puntaje

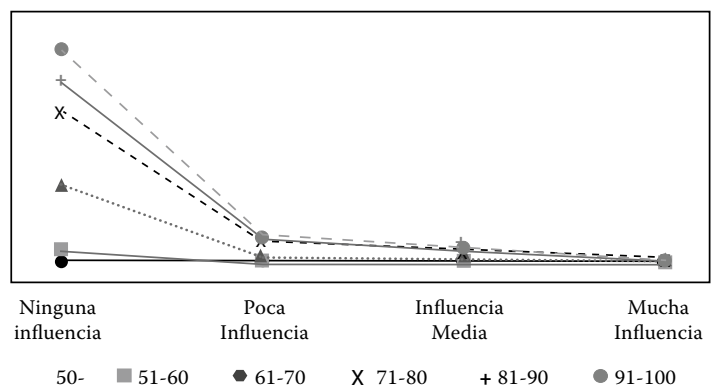

Fuente: elaboración propia.

Como en la Facultad de Ciencias, en artes la mayoría de estudiantes consideran que su puntaje en el componente de matemática tuvo poca o ninguna influencia en la decisión de su carrera, pero inesperadamente en esta facultad a mayor puntaje mayor percepción de los estudiantes que el Saber 11 tuvo influencia, esto podría contradecir la creencia que los estudiantes de artes eligen su carrera por falta de habilidades en otras áreas, como la matemática.

Gráfica 6. Tendencia de opción alternativa previa a la realización del examen Saber 11 en relación al puntaje (Artes)

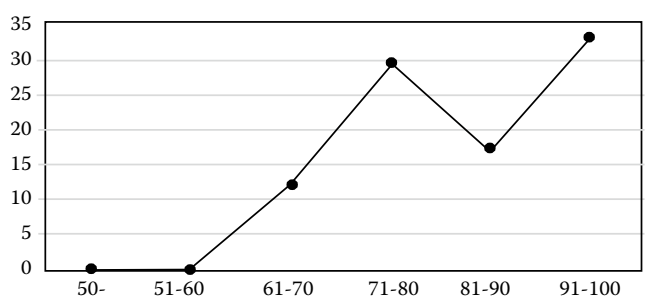

Fuente: elaboración propia.

En la Facultad de Artes, a mayor puntaje mayor era la elección de otra carrera. Para los autores, este resultado podría demostrar que la influencia del componente de matemáticas en la elección de carrera es nula, ya que los estudiantes de la Facultad de Artes tienen puntajes altos y estudian carreras que convencionalmente se consideran alejadas de la matemática. 
Tabla 3. Ciencias Económicas

\begin{tabular}{|l|l|c|}
\hline Facultad & \multicolumn{1}{|c|}{$\begin{array}{c}\text { Carreras a las } \\
\text { que se le aplicó la } \\
\text { encuesta }\end{array}$} & $\begin{array}{c}\text { Número de } \\
\text { encuestas } \\
\text { realizadas }\end{array}$ \\
\hline $\begin{array}{l}\text { Ciencias } \\
\text { Económicas }\end{array}$ & $\begin{array}{l}\text { Administración de } \\
\text { Empresas, Contaduría } \\
\text { Pública, Economía }\end{array}$ & \multicolumn{2}{|l}{} \\
\hline
\end{tabular}

Fuente: elaboración propia.

En la Facultad de Ciencias Económicas se aplicaron 60 encuestas. A continuación se presentarán las gráficas que arrojaron las encuestas y el análisis respectivo de cada una:

Gráfica 7. Número de personas en cada intervalo de puntaje (Ciencias Económicas)

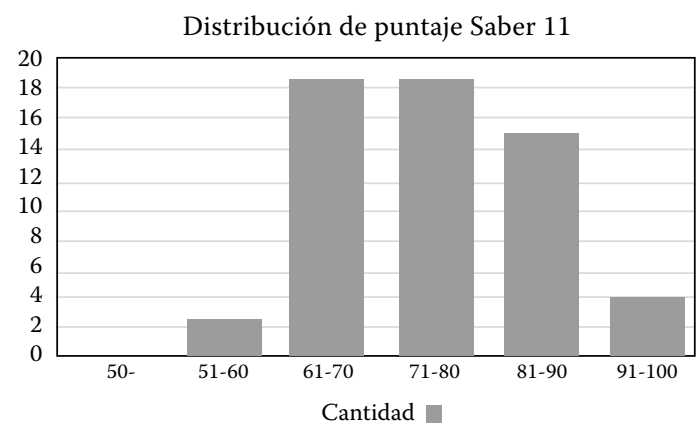

Fuente: elaboración propia.

Los estudiantes de la Facultad de Ciencias Económicas cuentan con un puntaje en matemáticas superior a 50, y la mayoría se ubica entre los rangos de 61 a 70, y de 71 a 80 (puesto que estos dos rangos tienen los mismos valores).

Gráfica 8. Tendencia de influencia por puntaje (Ciencias Económicas)

Tendencia de influencia por puntaje

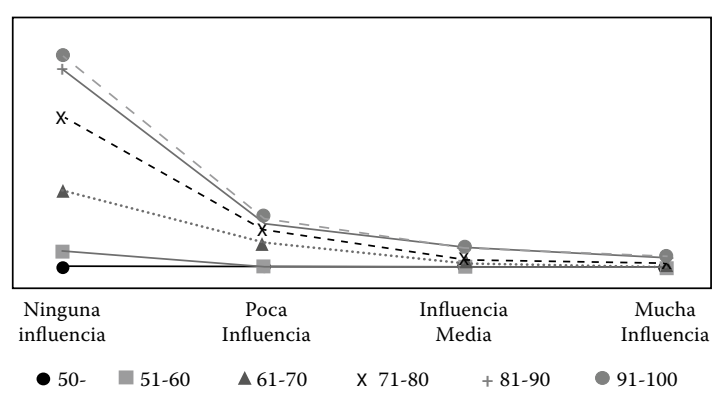

Fuente: elaboración propia.
Como se puede apreciar en la gráfica anterior, los estudiantes de la Facultad de Ciencias Económicas consideran que influyó poco el puntaje obtenido, sea cual sea el rango de puntaje en el que se encuentre.

Gráfica 9. Tendencia de opción alternativa previa a la realización del examen Saber 11 en relación al puntaje (Artes)

Opción de carrera previa al Saber 11

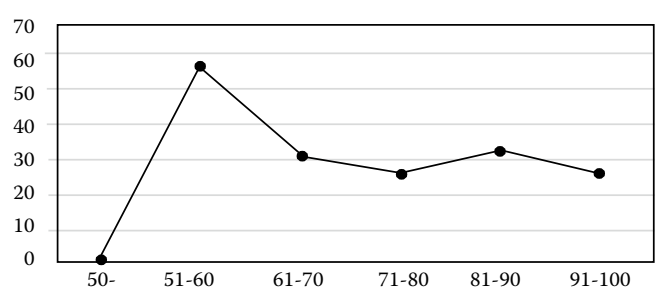

Fuente: elaboración propia.

Por último, es importante tener en cuenta las opciones de carrera de los estudiantes posterior a los resultados. En la siguiente grafica podemos apreciar que al igual que la influencia, independiente del rango, los estudiantes no contaban con otra opción. Y los que sí contaban con otra opción, se ubican mayormente en los rangos 61-70.

Tabla 4. Ciencias Humanas

\begin{tabular}{|l|l|c|}
\hline Facultad & $\begin{array}{l}\text { Carreras a las que se } \\
\text { le aplicó la encuesta }\end{array}$ & $\begin{array}{c}\text { Número de } \\
\text { encuestas } \\
\text { realizadas }\end{array}$ \\
\hline $\begin{array}{l}\text { Ciencias } \\
\text { Humanas }\end{array}$ & $\begin{array}{l}\text { Filosofía, Estudios } \\
\text { Literarios, Lingüística, } \\
\text { Español y Filología } \\
\text { Clásica, Filología } \\
\text { e Idiomas, Trabajo } \\
\text { Social, Antropología, } \\
\text { Sociología, Geografía, } \\
\text { Historia, Psicología }\end{array}$ & 47 \\
\hline
\end{tabular}

Fuente: elaboración propia.

En la Facultad de Ciencias Humanas se realizaron 47 encuestas a estudiantes. A continuación se presentarán las gráficas que arrojaron las encuestas y el análisis respectivo de cada una: 
Gráfica 10. Número de personas en cada intervalo de puntaje (Ciencias Humanas)

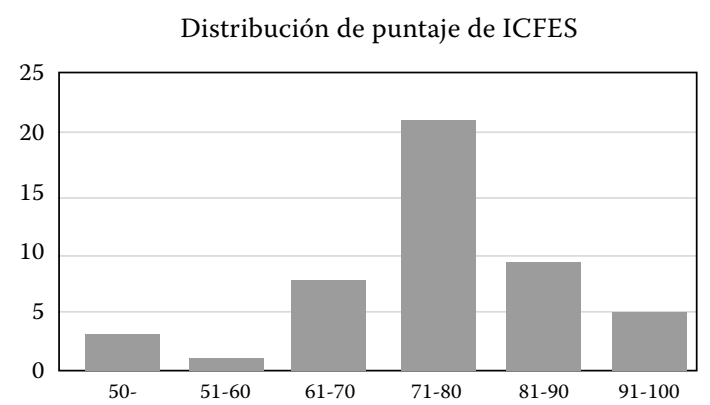

Fuente: elaboración propia.

En la Facultad de Ciencias Humanas de la Universidad Nacional de Colombia, sede Bogotá, los aspirantes, en su mayoría, obtuvieron puntajes en el componente de matemáticas entre 71 y 90 puntos. Siendo este considerado un puntaje alto evidenciando que por ser una facultad de ciencias humanas no necesariamente las aptitudes matemáticas de los aspirantes son bajas.

Gráfica 11. Tendencia de influencia por puntaje (Ciencias Humanas)

Tendencia de influencia por puntaje

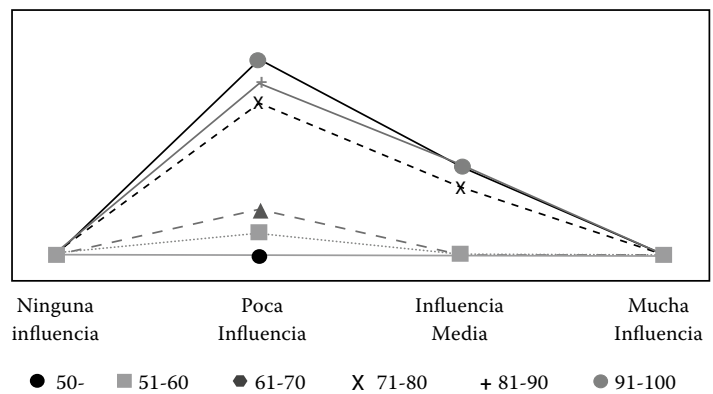

Fuente: elaboración propia.

Siguiendo la tendencia de las anteriores facultades, en ciencias humanas los estudiantes consideran que su puntaje en el componente de matemática en la prueba Saber 11 tuvo o poco o ninguna influencia en el momento de elegir la carrera. De la misma manera, entre más puntaje más se considera que el puntaje fue irrelevante para la decisión de qué estudiar.
Tabla 5. Derecho y Ciencias Políticas

\begin{tabular}{|l|l|c|}
\hline \multicolumn{1}{|c|}{ Facultad } & $\begin{array}{c}\text { Carreras a las } \\
\text { que se le aplicó } \\
\text { la encuesta }\end{array}$ & $\begin{array}{c}\text { Número de } \\
\text { encuestas } \\
\text { realizadas }\end{array}$ \\
\hline $\begin{array}{l}\text { Derecho y } \\
\text { ciencias políticas }\end{array}$ & $\begin{array}{l}\text { Derecho y } \\
\text { Ciencias Políticas }\end{array}$ & 52 \\
\hline
\end{tabular}

Fuente: elaboración propia.

En la Facultad de Derecho y Ciencias Políticas se realizaron 52 encuestas a estudiantes. A continuación se presentarán las gráficas que arrojaron las encuestas y el análisis respectivo de cada una:

Gráfica 12. Número de personas en cada intervalo de puntaje (Derecho y Ciencias Políticas)

Distribución de puntaje de ICFES

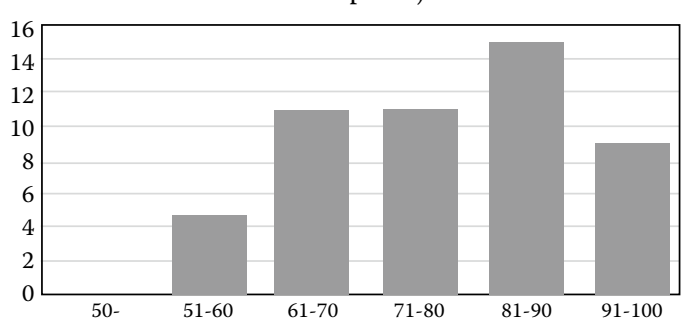

Fuente: elaboración propia.

Se encontró que el puntaje promedio que obtuvieron los encuestados está entre 81-90, y que el mismo lo obtuvieron en su mayoría estudiantes de ciencias políticas, y que a pesar de tener un puntaje considerado alto en la prueba saber 11 (ICFES) decidieron escoger una carrera que no posee un alto contenido matemático.

Gráfica 13. Tendencia de influencia por puntaje (Derecho y Ciencias Políticas)

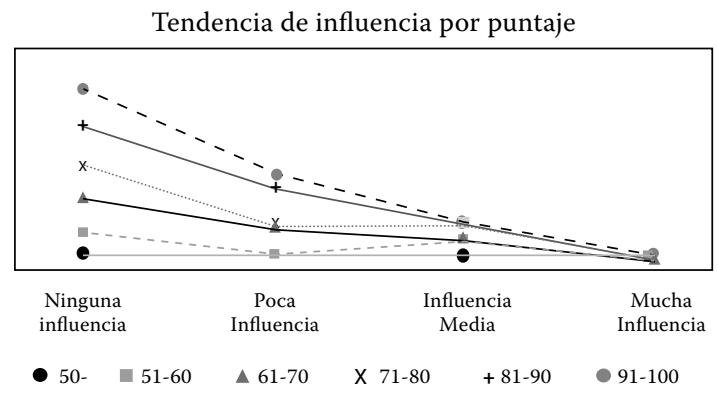

Fuente: elaboración propia. 
Se puede identificar que independientemente del puntaje que los estudiantes sacaron en el componente de matemáticas, alrededor del $54 \%$ de la población encuestada respondió que el mismo no tiene ninguna influencia frente a la decisión de escoger carrera universitaria.

Gráfica 14. Tendencia de opción alternativa previa a la realización del examen Saber 11 en relación al puntaje (Derecho y Ciencias Políticas)

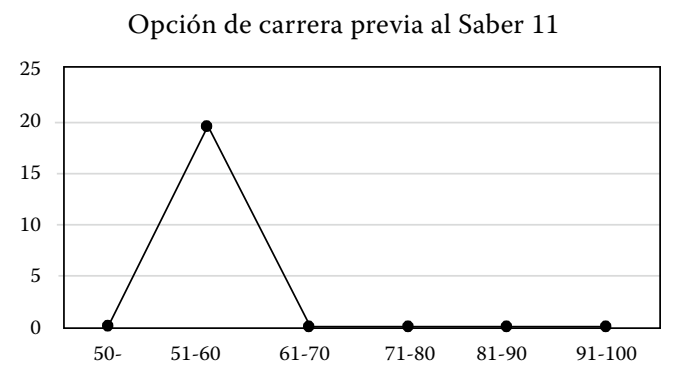

Fuente: elaboración propia.

Con relación a la posibilidad de realizar una segunda carrera, y como esto depende del resultado del componente matemático de las pruebas saber, se puede inferir que este último no tiene ningún peso en esta importante decisión, sino que se continúa manteniendo la tendencia de elegir por motivos subjetivos.

Tabla 6. Enfermería

\begin{tabular}{|c|l|l|}
\hline Facultad & $\begin{array}{c}\text { Carreras a las } \\
\text { que se le aplicó } \\
\text { la encuesta }\end{array}$ & $\begin{array}{c}\text { Número de } \\
\text { encuestas } \\
\text { realizadas }\end{array}$ \\
\hline Enfermería & Enfermería & 60 \\
\hline
\end{tabular}

Fuente: elaboración propia.

En la Facultad de Enfermería se realizaron 60 encuestas a los estudiantes. A continuación se presentarán las gráficas que arrojaron las encuestas y el análisis respectivo de cada una:
Gráfica 15. Número de personas en cada intervalo de puntaje (Enfermería)

Distribución de puntaje de ICFES

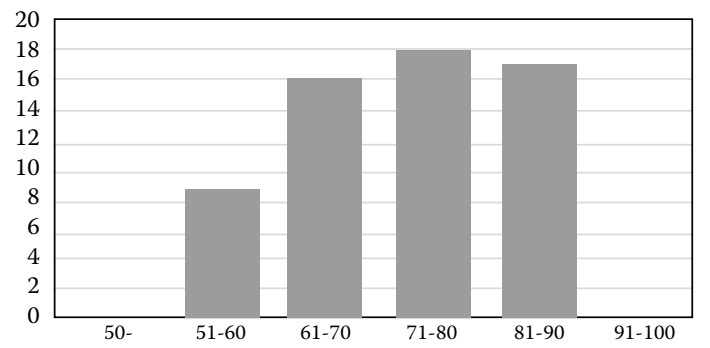

Fuente: elaboración propia.

El presente gráfico muestra que, en esta facultad, la mayoría de los encuestados obtuvieron un puntaje promedio de entre 71 y 80 puntos. Esto quiere decir que, en promedio, los estudiantes de esta facultad estuvieron entre algunos de los percentiles más altos en el componente matemático de la prueba de estado.

Gráfica 16. Tendencia de influencia por puntaje (Enfermería)

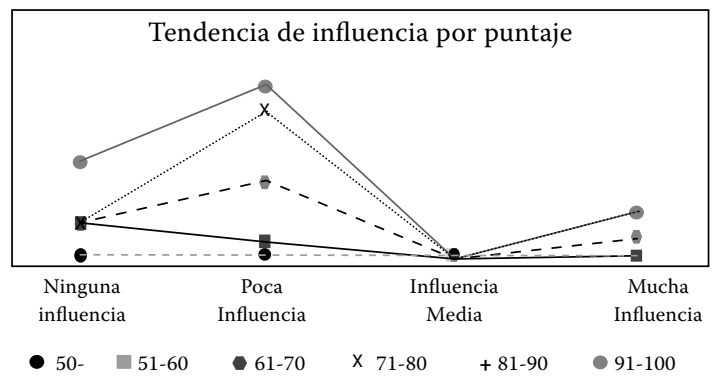

Fuente: elaboración propia.

La segunda gráfica corrobora las conclusiones ya expuestas anteriormente. Según esta, el ICFES tuvo poca o ninguna influencia para la mayoría de los estudiantes en todas las categorías, presentando unas cuantas excepciones para los estudiantes que se encuentran en los rangos de puntuación entre 51-60 y 61-70 puntos. Cabe resaltar que los casos en los que el ICFES jugó un rol importante fueron la minoría, e incluso entre los puntajes más bajos, la mayoría de los estudiantes reportó una baja correlación entre el puntaje y la carrera escogida. 
Se puede evidenciar a partir de la gráfica que el grado de influencia del resultado del ICFES en la decisión de carrera es un poco mayor en los puntajes de rangos medios (61-70 y 71-80) que en los puntajes de rangos extremos (51-60 y 81-90), sin que esto quiera decir que en la mayoría de los casos el ICFES tuviese mucha influencia en las decisiones de la carrera que escogieron los estudiantes en general.

Gráfica 17. Tendencia de opción alternativa previa a la realización del examen Saber 11 en relación al puntaje (Enfermería)

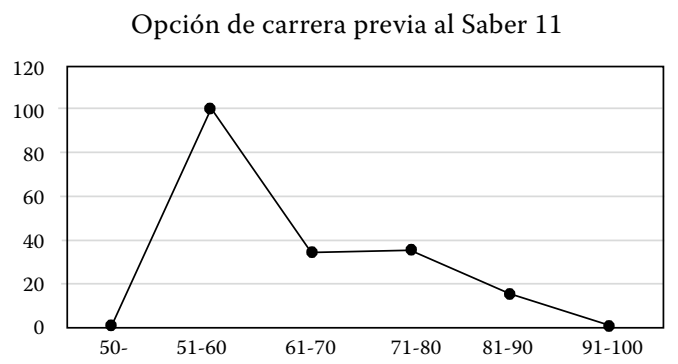

Fuente: elaboración propia.

Finalmente, en la Facultad de Enfermería, entre más puntaje más seguridad de la carrera escogida. Podríamos afirmar que esto se debe a que en la Facultad de Enfermería la cantidad de materias con componentes matemáticos es superior a la de otras facultades; por consiguiente, los estudiantes se sienten más seguros si tienen mayor puntaje.

Tabla 7. Ingeniería

\begin{tabular}{|l|l|l|}
\hline Facultad & $\begin{array}{l}\text { Carreras a las que se le } \\
\text { aplicó la encuesta }\end{array}$ & $\begin{array}{l}\text { Número de } \\
\text { encuestas } \\
\text { realizadas }\end{array}$ \\
\hline Ingeniería & $\begin{array}{l}\text { Ingeniería Agrícola, } \\
\text { Ingeniería Civil, } \\
\text { Ingeniería de Sistemas y } \\
\text { Computación, Ingeniería } \\
\text { Eléctrica, Ingeniería } \\
\text { Electrónica, Ingeniería } \\
\text { Industrial, Ingeniería } \\
\text { Mecánica, Ingeniería } \\
\text { Mecatrónica, Ingeniería } \\
\text { Química }\end{array}$ & \\
\hline
\end{tabular}

Fuente: elaboración propia.
Distribución del puntaje de la prueba saber 11: En la Facultad de Ingeniería se realizaron 54 encuestas a estudiantes. A continuación se presentarán las gráficas que arrojaron las encuestas y el análisis respectivo de cada una.

Gráfica 18. Número de personas en cada intervalo de puntaje (Ingeniería)

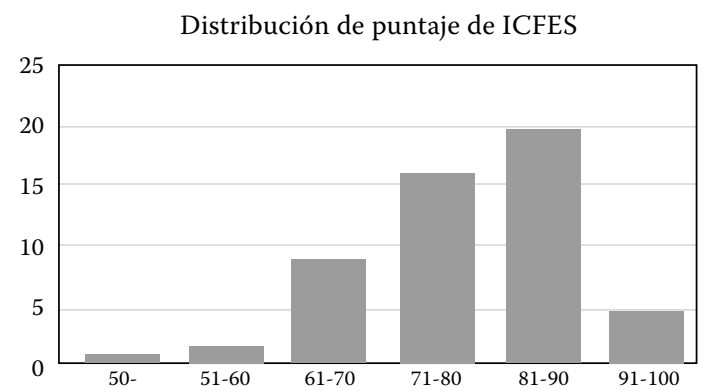

Fuente: elaboración propia.

La gráfica ilustra una clara tendencia hacia la derecha, indicando que el puntaje matemático de la facultad tiende a ser elevado. Esto concuerda con la hipótesis que dice que a mayor puntaje, mayor la tendencia a elegir una carrera convencionalmente orientada hacia las matemáticas.

Gráfica 19. Tendencia de influencia por puntaje (Ingeniería)

Tendencia de influencia por puntaje

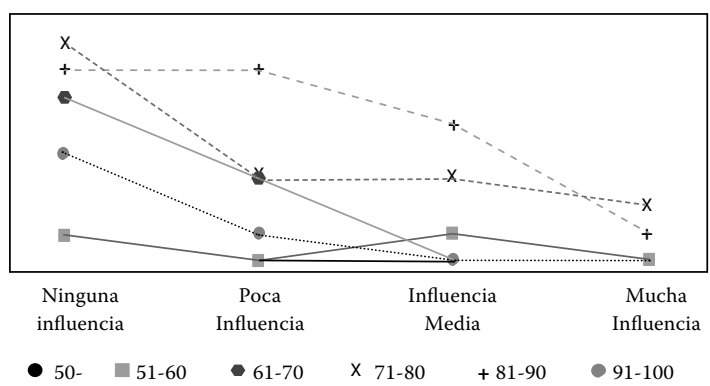

Fuente: elaboración propia.

Esta gráfica muestra que, por lo general, los alumnos de la Facultad de Ingeniería no consideraron el puntaje como un factor influyente en la decisión de su carrera. 
Gráfica 20. Tendencia de opción alternativa previa a la realización del examen Saber 11 en relación al puntaje (Ingeniería)

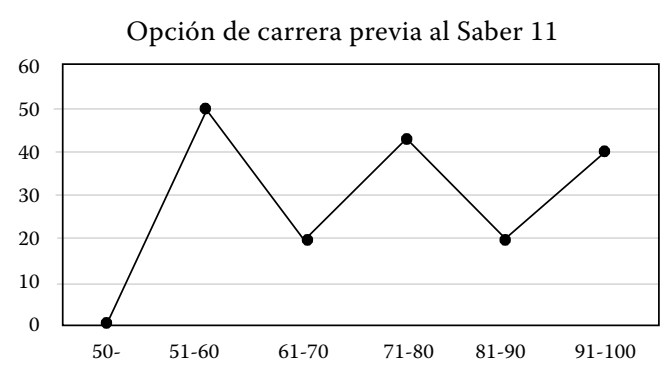

Fuente: elaboración propia.

La gráfica ilustra que no hay relación que se pueda inferior a simple vista, el puntaje no afecta en la elección final de la carrera.

\section{Análisis de la Universidad}

Gráfica 21. Número de personas en cada intervalo de puntaje (Universidad)

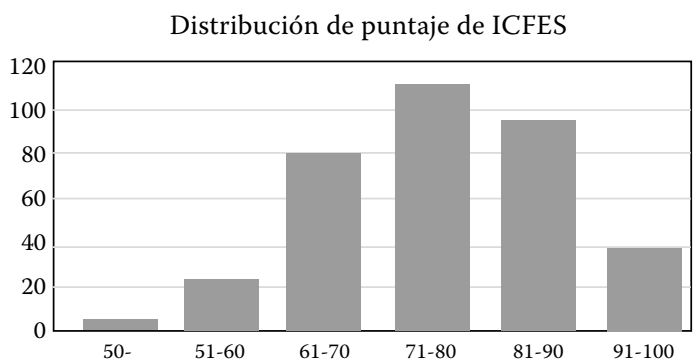

Fuente: elaboración propia.

De la gráfica se puede identificar el desplazamiento horizontal hacia la derecha del acumulado de puntaje de ICFES en el componente de matemáticas que poseen los estudiantes de la Universidad Nacional de Colombia. De entrada se demuestra el alto nivel matemático de la Universidad, estando la mayoría de los encuestados en el rango de 71-80 o superior.
Gráfica 22. Tendencia de influencia por puntaje (Universidad)

Tendencia de influencia por puntaje

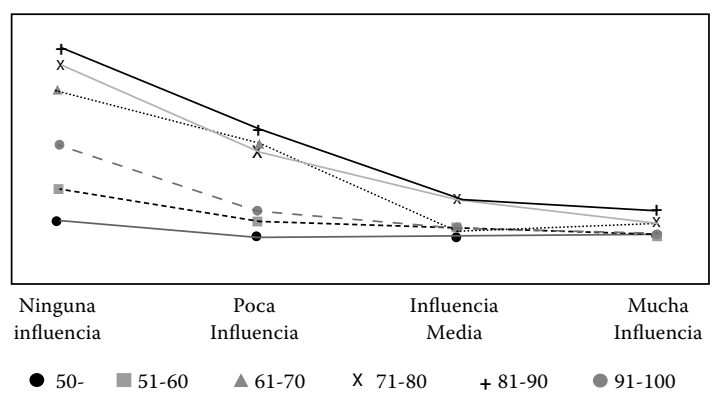

Fuente: elaboración propia.

Esta grafica muestra de manera contundente la poca relevancia, en general, del resultado matemático en el examen Saber 11 al momento de elegir una carrera. Se destaca, sin embargo, que aquellos donde la influencia fue más elevada se encuentran principalmente en el rango de 50 puntos o menos en el examen Saber 11. De un modo similar se puede observar que aquellos donde el nivel matemático tuvo menor relevancia en la decisión se encuentran primordialmente ubicados en los rangos superiores de los puntajes.

Gráfica 23. Tendencia de opción alternativa previa a la realización del examen Saber 11 en relación al puntaje (Universidad)

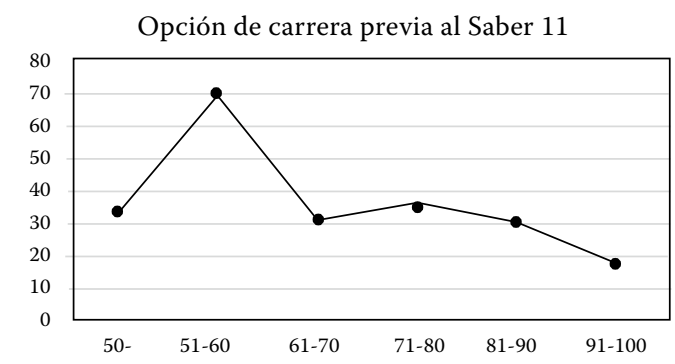

Fuente: elaboración propia.

Ilustrada en la gráfica se ve la tendencia a poseer una carrera alternativa a la carrera con la cual se ingresó en los rangos inferiores del puntaje ICFES en el componente de matemáticas. Aquellos con mayor puntaje demuestran menos incertidumbre en la escogencia de su carrera. 


\section{Conclusiones}

Analizando en su conjunto los resultados de las encuestas se han ido formando unas conclusiones que, en su totalidad, vienen a refutar nuestra hipótesis principal y comprobar nuestra hipótesis secundaria.

En primer lugar se puede afirmar, de manera contundente, que la influencia del nivel matemático en la elección de carrera del estudiante de la Universidad Nacional de Colombia es notoriamente baja independientemente de la facultad de ingreso, pues la variación de la influencia percibida entre facultades es de un valor mínimo. Quizá la poca relevancia otorgada al resultado se debe a la naturaleza de entrada a la Universidad Nacional de Colombia, donde el resultado más importante no es el del ICFES sino el del examen de ingreso. La alta demanda por cupos en la universidad propone una fuerte competencia entre aspirantes, de esto modo se propondría que, a menor puntaje, menor la posibilidad de ingreso a la universidad para la carrera de preferencia. Esto a su vez explica el por qué el estudiante de menor puntaje tiende a contar con una alternativa de carrera en caso tal de no ser admitido a su opción inicial.

En coherencia con esto es posible proponer que el alto nivel de competencia opera como un filtro, donde los aspirantes, conscientes del alto nivel de competencia, o cuentan con una aptitud natural para las matemáticas que les aumente la probabilidad de ingreso a la universidad, o son excluidos de la población de admitidos incluso antes de presentar el examen de ingreso dados sus puntajes básicos en el examen nacional Saber 11.

Es por esto que, independientemente de la carrera elegida, el nivel matemático del cuerpo estudiantil es de por sí elevado, implicando que la influencia del nivel matemático se verá reducida, puesto que se espera, de antemano, que el aspirante a la Universidad Nacional de Colombia tenga una capacidad matemática significativa con la cual competir por su puesto de admisión.

\section{Referencias}

Cégep L. (2012). Tomado de: https://www. cegeplimou.ca/media/all/59609/Fiche-carrieres-en-Sciences-de-la-nature.pdf

Dijk, T. (2001). La ciencia del texto. Barcelona: Paidós.

Harkins, M., \& Singer, S. (2009). The Conundrum of Large Scale Standardized Testing: Making Sure Every Student Counts. Journal of Thought, 44(1-2), 77. http://dx.doi.org/10.2307/ jthought.44.1-2.77

Hernández, R., et al. (2014). Metodología de la investigación. México: McGraw-Hill Education.
Ortiz, M., y Zabala, A. (2001). Las actitudes y su influencia en el desempeño de los estudiantes en área de matemáticas. Caracas: Corporación Educativa Mayor del Desarrollo Simón Bolivar, pp. 76-80. Tomado de https:// hera.ugr.es/tesisugr/2108144x.pdf

Ortiz, M. y Zabala, J. (2005). Las actitudes y su influencia en el desempeño de los estudiantes en el área de matemáticas. 01/10/2016, de Colombia aprende Sitio web: www.colombia aprende.edu.co/html/docentes/1596/articles104886_archivo.doc 


\section{Anexo 1. Encuesta aplicada}

\section{Influencia del puntaje ICFES en el área de matemática en la elección de carrera.}

Objetivo: identificar la influencia de los resultados de la prueba de estado en la elección de carrera al momento de ingresar a la universidad.

1. Facultad:

\section{Carrera:}

3. Marque con una $\mathrm{X}$ el rango en el que se encuentra su puntaje de la prueba Saber 11 en el área de matemática:

3.1 _ Menos de 50 puntos.

3.2 _ Entre 51 puntos y 60 puntos.

3.3 _ Entre 61 puntos y 70 puntos.

$3.4 \ldots$ Entre 71 puntos y 80 puntos.

3.5 _ Entre 81 puntos y 90 puntos.

$3.6 \_$Entre 91 puntos y 100 puntos.
4. ¿Está entre sus planes realizar un doble programa con una carrera que no pertenece a su facultad?
$4.1 \_$Sí
4.2 _ No

4.3 Si la respuesta anterior fue afirmativa: escriba cuál la otra opción de carrera:

5. ¿Qué influencia tuvo su puntaje del ICFES en la elección de la carrera que actualmente estudia?
5.1 _ Nada de influencia.
5.2 _ Poca influencia.
5.3 _ Influencia media.
5.4 _ Mucha influencia.

6. ¿Antes de saber el resultado del ICFES tenía otra opción de carrera?
$6.1-$ No
6.2 - Sí

6.3 Si la respuesta anterior fue afirmativa: escriba cuál la otra opción de carrera:

\section{Anexo 2. Muestras poblacionales recopiladas por las encuestas}

\begin{tabular}{|c|c|c|}
\hline Facultad & Carreras a las que se le aplicó la encuesta & $\begin{array}{l}\text { Número de } \\
\text { encuestas realizadas }\end{array}$ \\
\hline Ciencias & Matemáticas, Estadística, Física, Química, Biología. & 52 \\
\hline Artes & Diseño industrial. & 32 \\
\hline $\begin{array}{l}\text { Ciencias } \\
\text { Económicas }\end{array}$ & $\begin{array}{l}\text { Administración de Empresas, Contaduría Pública, } \\
\text { Economía }\end{array}$ & 60 \\
\hline Ciencias Humanas & $\begin{array}{l}\text { Filosofía, Estudios Literarios, Lingüística, Español y } \\
\text { Filología Clásica, Filología e Idiomas, Trabajo Social, } \\
\text { Antropología, Sociología, Geografía, Historia, Psicología }\end{array}$ & 47 \\
\hline $\begin{array}{l}\text { Derecho y ciencias } \\
\text { políticas }\end{array}$ & Derecho y ciencias políticas & 52 \\
\hline Enfermería & Enfermería & 60 \\
\hline Ingeniería & $\begin{array}{l}\text { Ingeniería Agrícola, ingeniería Civil, Ingeniería de } \\
\text { Sistemas y Computación, Ingeniería Eléctrica, Ingeniería } \\
\text { Electrónica, Ingeniería Industrial, Ingeniería Mecánica, } \\
\text { Ingeniería Mecatrónica, Ingeniería Química }\end{array}$ & 54 \\
\hline
\end{tabular}

